\title{
Beweis, dass eine für jeden reellen Werth von $x$ durch eine trigonometrische Reihe gegebene Function $f(x)$ sich nur auf eine einzige $W$ eise in dieser Form darstellen lässt.
}

(Von Herrn G. Cantor in Halle.)

Wenn eine Function $f(x)$ einer reellen Veränderlichen $x$ durch eine für jeden Werth von $x$ convergente, trigonometrische Reihe:

$$
f(x)=\frac{1}{2} b_{0}+\left(a_{1} \sin x+b_{1} \cos x\right)+\cdots+\left(a_{n} \sin n x+b_{n} \cos n x\right)+\cdots
$$

gegeben ist, so ist es von Wichtigkeit, zu wissen, ob es noch andre Reihen von derselben Form giebt, welche ebenfalls für jeden Werth von $x$ convergiren und die Function $f(x)$ darstellen. Diese Frage, welche erst in neuester Zeit angeregt worden ist, kann nicht etwa, wie gewöhnlich angenommen wird, dadurch entschieden werden, dass man jene Gleichung mit $\cos n(x-t) d x$ multiplicirt und Glied für Glied von $-\pi$ bis $+\pi$ integrirt (wobei in der That auf der rechten Seite nur das aus dem $n^{\text {ten }}$ Gliede hervorgehende Integral nicht wegfallen würde); denn, abgesehen davon, dass hierbei die Möglichkeit der Integration von $f(x)$ vorausgesetzt würde, kann die Integration einer Reihe:

$$
A_{0}+A_{1}+\cdots+A_{n}+\cdots
$$

in welcher die $A_{n}$ Functionen einer Veränderlichen $x$ sind, durch Integration ihrer Theile nur dann ohne Bedenken ausgeführt werden, wenn der Rest, welcher nach Abtrennung der $n$ ersten Glieder übrig bleibt, für alle Werthe von $x$, welche im Integrationsintervalle liegen, gleichzeitig unendlich klein wird. Also muss, wenn man:

$$
f(x)=A_{0}+A_{1}+\cdots+A_{n}+R_{n}
$$

setzt, bei gegebener Grösse $\varepsilon$, eine ganze Zahl $m$ vorhanden sein, so beschaffen, dass für $n \sum m, \boldsymbol{R}_{n}$ seinem absoluten Betrage nach kleiner ist als $\varepsilon$ für alle Werthe von $x$, welche in Betracht kommen.

Es ist nämlich die kleinste ganze Zahl $m$, welche für ein gegebenes $\boldsymbol{x}$ die Bedingung erfüllt, dass der absolute Betrag von $\boldsymbol{R}_{n}$ kleiner ist als $\varepsilon$,

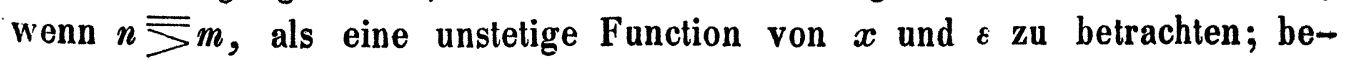
18 * 
zeichnet man sie unter dinscn Umständen genauer mit $m(x, \varepsilon)$, so weiss man nicht, ob die Furifi si $m(x, \varepsilon)$ bei gegebenem $\varepsilon$ für alle Werthe von $x$ unterhalb einer endlichen Grenze liegt; es ist sogar leicht einzusehen, dass, wenn $f(x)$ für $x=x_{1}$ eine Unstetigkeit hat, die Function $m(x, \varepsilon)$ Werthe annehmen muss, welche jede angebbare Grenze übersteigen, wenn, bei festgehaltenem $\varepsilon, x$ dem Werthe $x_{1}$ unendlich nahe rückt.

Hieraus geht hervor, dass die Eindeutigkeit der Darstellung einer Function durch eine für jeden Werth von $x$ convergente trigonometrische Reihe auf diesem Wege nicht ergründet werden kann.

Durch die Riemannsche Abhandlung "Ueber die Darstellung einer Function durch eine trigonometrische Reihe, Göttingen 1867" bin ich auf einen andern, das Ziel erreichenden Weg geführt worden, welchen ich hier kurz angeben will.

Zuerst hebe ich hervor, dass, wie in meinem Aufsatze „Ueber einen die trigonometrischen Reihen betreffenden Lehrsatz" bewiesen ist, bei einer trigonometrischen Reihe:

$$
A_{0}+A_{1}+\cdots+A_{n}+\cdots,
$$

welche für sämmtliche Werthe von $x$ in einem gegebenen, übrigens beliebig kleinen Intervalle des reellen Grössengebietes convergirt, die Coefficienten $a_{n}, b_{n}$ mit wachsendem $n$ unendlich klein werden.

Denkt man sich nun zwei trigonometrische Reihen, welche für jeden reellen Werth von $x$ convergiren und denselben Werth annehmen, mithin dieselbe Function $f(x)$ darstellen, so folgt durch Abziehen der einen von der andern, eine für jeden Werth von $x$ convergente Darstellung der Null:

$$
\text { (1.) } 0=C_{0}+C_{1}+\cdots+C_{n}+\cdots
$$

wo $C_{0}=\frac{1}{2} d_{0}, C_{n}=c_{n} \sin n x+d_{n} \cos n x$ und wo die Coefficienten $c_{n}, d_{n}$ mit wachsendem $n$, nach dem soeben Gesagten, unendlich klein werden. Ich bilde mit Riemann aus der Reihe (1.) die Function:

$$
\text { (2.) } \quad F(x)=C_{0} \frac{x x}{2}-C_{1}-\cdots-\frac{C_{n}}{n n}-\cdots
$$

Sie ist eine in der Nähe eines jeden Werthes von $x$ stetige Function von $x^{*}$ ), welche dem Lehrsatze 1 im $\$$. 8 der vorhin genannten Abhandlung zufolge, die Eigenschaft hat, dass für jeden Werth von $x$ der zweite Differenzquotient:

*) Um dies einzusehen, wỉrde es ausreichen, wenn man nur wüsste, dass die $c_{n}, d_{n}$ unter einer angebbaren Grenze liegen, der Nachweis davon dürfte jedoch dieselben Mittel in Anspruch nehmen, mit welchen gezeigt wird, dass $\lim c_{n}=0$ und $\lim d_{n}=0$. 


$$
\frac{F(x+\alpha)-2 F(x)+F(x-\alpha)}{\alpha \alpha}
$$

mit unendlich abnehmendem $\alpha$ sich der Grenze Null nähert.

Halțen wir diese beiden data für die Function $F(x)$ fest:

I. dass sie stetig ist in der Nähe eines jeden Werthes von $x$,

II. dass die Grenze ihres zweiten Differenzquotienten mit unendlich abnehmendem $\alpha$ für jeden Werth von $x$ gleich Null ist, so lässt sich daraus zeigen, dass $F(x)$ eine ganze Function ersten Grades $c x+c^{\prime}$ ist. Der folgende Beweis hiervon ist mir von Herrn Schwarz in Zürich mitgetheilt worden *).

$M$ an denke sich bei einer in einem Intervalle $(a \ldots b)$ der reellen Veränderlichen $x$ gegebenen Function $F_{1}(x)$ die Bedingungen I. und II. erfüllt, und zwar die erste in der Nähe eines jeden Werthes von $x$ im Intervalle, die zweite für jeden $\mathrm{Zwischenwerth} x$, und betrachte, indem man unter $i$ die positive oder negative Einheit, unter $\%$ irgend eine reelle Grösse versteht, die Function:

$$
\varphi(x)=i\left\{F_{1}(x)-F_{1}(a)-\frac{x-a}{b-a}\left(F_{1}(b)-F_{1}(a)\right)\right\}-\frac{x^{2}}{2}(x-a)(b-x) .
$$

Aus den Voraussetzungen über $F_{1}(x)$ folgt, dass $\varphi(x)$ im Intervalle $(a \ldots b)$ stetig ist, und dass die Grenze des zweiten Differenzquotienten von $\varphi(x)$ gleich $\varkappa^{2}$ ist für jeden Zwischenwerth $x$, bei unendlich abnehmendem $\alpha$; ferner ist : $\varphi(a)=0, \varphi(b)=0$. Bezeichnen wir daher:

$$
\varphi(x+\alpha)-2 \varphi(x)+\varphi(x-\alpha) \text { mit } \varphi(x, \alpha),
$$

so ist $\varphi(x, \alpha)$ für jeden Zwischenwerth $x$, bei unendlich abnehmendem $\alpha$, annähernd gleich $\varkappa^{2} \alpha^{2}$, also positiv und von Null verschieden für hinreichend kleine Werthe von $\alpha$. Daraus folgt, dass $\varphi(x)$ für keinen Werth von $x$ im Intervalle positiv ist. In der That an den Grenzen ist $\varphi(x)=0$; würde $\varphi(x)$ für einen Zwischenwerth positiv sein, so wäre das Maximum der Werthe, welche $\varphi(x)$ annehmen kann, ebenfalls eine positive Grösse und würde zum Mindesten für einen Zwischenwerth $x_{0}$ von $x$ erreicht; es wäre also für hinreichend kleine

*) Dieser Beweis stützt sich im Wesentlichen auf den in den Vorlesungen des Herrn Weierstrass häufig vorkommenden und bewiesenen Satz:

,Eine in einem Intervalle $(a \ldots b)$ (die Grenzen incl.) der reellen Veränderlichen $x$ gegebene, stetige Function $\varphi(x)$ erreicht das Maximum $g$ der Werthe, welche sie annehmen kann, zum Mindesten für einen Werth $x_{0}$ der Veränderlichen, so dass $\varphi\left(x_{0}\right)=g$.

Einen ähnlichen, auch hierauf beruhenden Beweis für den Fundamentalsatz der Differentialrechnung hat Ossian Bonnet gefuhrt; derselbe findet sich in „Cours de calcul différentiel et intégral, par J. A. Serret, Paris $1868^{\star}$ im ersten Bande, Seite 17-19. 
Werthe von $\alpha: \varphi\left(x_{0}+\alpha\right)-\varphi\left(x_{10}\right) \bar{\Sigma} 0, \varphi\left(x_{10}-\alpha\right)-\varphi\left(x_{10}\right) \bar{\sum}$, mithin auch: $\varphi\left(x_{0}, \alpha\right) \overline{\bar{\alpha}}$, während doch $\varphi\left(x_{0}, \alpha\right)$ für hinreichend kleine Werthe von $\alpha$ positiv ist. Man hat also für jeden Werth von $x$ im Intervalle $(a \ldots b)$, für $i= \pm 1$ und für einen beliebigen reellen Werth von $\%$ :

$$
\varphi(x) \equiv 0 \text {; }
$$

lässt man hierin $\varkappa$ unendlich klein werden, so folgt:

$$
F_{1}(x)=F_{1}(a)+\frac{x-a}{b-a}\left(F_{1}(b)-F_{1}(a)\right) .
$$

Es ist also unter den gemachten Voraussetzungen $F_{1}(x)$ eine ganze Function ersten Grades von $x$.

Daraus ergiebt sich für unsere Function $\boldsymbol{F}(\boldsymbol{x})$ (da hier das Intervall beliebig erweitert werden kann) die für alle Werthe von $x$ gültige Form: $\boldsymbol{F}(\boldsymbol{x})=\boldsymbol{c} \boldsymbol{x}+\boldsymbol{c}^{\prime}$, und man hat daher für jeden Werth von $\boldsymbol{x}$ :

$$
C_{0} \frac{x x}{2}-c x-c^{\prime}=C_{1}+\frac{C_{2}}{4}+\cdots+\frac{C_{n}}{n n}+\cdots
$$

Aus der Periodicität auf der rechten Seite ergiebt sich zunächst, dass sowohl $c=0$, wie auch $C_{0}=\frac{d_{0}}{2}=0$, und man behält daher die Gleichung:

$$
\text { (3.) }-c^{\prime}=C_{1}+\frac{C_{2}}{4}+\cdots+\frac{C_{n}}{n n}+\cdots \cdot
$$

Die Reihe rechts ist von der Art, dass man, bei gegebenem $\varepsilon$, eine ganze Zahl $m$ angeben kann, so dass, wenn $n \equiv m$, der Rest $R_{n}$ seinem absoluten Betrage nach kleiner als $\varepsilon$ ist für alle Werthe von $x$.

Man kann daher die Gleichung (3.) nach Multiplication mit $\cos n(x-t) d x$ Glied für Glied von $-\pi$ bis $+\pi$ integriren; das Resultat ist

$$
c_{n} \sin n t+d_{n} \cos n t=0 \text {, }
$$

wo unter $t$ eine beliebige reelle Grösse zu verstehen ist; man hat also: $c_{n}=0$, $d_{n}=0$, während schon vorher gefolgert wurde, dass $d_{0}=0$.

Es ergiebt sich also das Resultat, dass eine für jeden einzelnen reellẹn Werth von $x$ convergente Darstellung der Null durch eine trigonometrische Reihe (1.) nicht anders möglich ist, als wenn die Coefficienten $d_{0}, c_{n}, d_{n}$ sämmtlich gleich Null sind und man hat den Satz:

;Wenn eine Function $f(x)$ einer reellen Veränderlichen $x$ durch eine für jeden Werth von $x$ convergente trigonometrische Reihe gegeben ist, so giebt es keine andere. Reihe von derselben Form, welche ebenfalls für jeden Werth von $x$ convergirt und die nämliche Function $f(x)$ darstellt."

Berlin, den 6. April 1870. 